\title{
SÍNTESE E REATIVIDADE DE SÍLICA LAMELAR
}

\author{
Robson Fernandes de Farias* \\ Departamento de Química - Universidade Federal de Roraima - 69310-270 - Boa Vista - Roraima. \\ Claudio Airoldi \\ Instituto de Química - Universidade Estadual de Campinas - CP 6154 - 13083-970 - Campinas - SP
}

Recebido em 2/9/98; aceito em 17/3/99

\begin{abstract}
SYNTHESIS AND REACTIVITY OF LAMELLAR SILICA. An overview of the experimental procedures to prepare lamellar samples of silica, as well as the reactivity and possible applications of this kind of material is presented. Special attention is focused on the obtained materials by using neutral dialkylamine route through sol-gel process.
\end{abstract}

Keywords: lamellar silica; sol-gel process; neutral diamine.

\section{INTRODUÇÃO}

\section{Sílica-Gel: Aspectos gerais}

Depois do oxigênio, o silício é o elemento mais abundante na crosta terrestre, ocorrendo ainda como elemento traço em muitos tecidos animais e vegetais, chegando a ser um elemento essencial para alguns organismos vivos. A fase mineral é sempre a sílica amorfa, com diferentes graus de hidratação, sendo representada por $\mathrm{SiO}_{2} \cdot \mathrm{nH}_{2} \mathrm{O}^{1}$.

A sílica-gel é um polímero inorgânico, consistindo de grupos siloxanos ( $\mathrm{Si}-\mathrm{O}-\mathrm{Si}$ ) em seu interior, e de grupos silanóis ( $\mathrm{Si}$ $\mathrm{OH})$ em sua superfície ${ }^{2}$, sendo a Stishovita seu único polimorfo não patogênico ${ }^{3-5}$.

Desde que Kiselev em 1936 descobriu a presença dos grupos hidroxilas na superfície da sílica ${ }^{6}$, muitos estudos foram desenvolvidos com o intuito de se calcular o número de grupos silanóis por unidade de área em sua superfície $\left(\alpha_{\mathrm{OH}} / \mathrm{OH}\right.$ $\left.\mathrm{nm}^{-2}\right)$. Zhuravlev ${ }^{7}$ estudando uma centena de diferentes amostras de sílica, através do método de troca de deutério acoplado a espectrometria de massa, concluiu que $\alpha_{\mathrm{OH}}$ é aproximadamente igual a $5,0 \mathrm{OH} \mathrm{nm}{ }^{-2}$, independentemente da origem ou das diferentes características estruturais, tais como área superficial, tipos de poros, tamanhos de poros, empacotamento das partículas e estrutura do esqueleto $\mathrm{SiO}_{2}$ da sílica analisada, chegando a considerar o valor de $\alpha_{\mathrm{OH}}$ como sendo uma constante físico-química. Recentemente ${ }^{8}$, utilizando-se termogravimetria (TG), chegou-se também a um valor médio de 5,0 $\mathrm{OH} \mathrm{nm}{ }^{-2}$ para a composição superficial da sílica.

A reatividade química da superfície da sílica é determinada principalmente pelos grupos hidroxilas (silanóis), os quais são fracamente ácidos ${ }^{9} \operatorname{com} \mathrm{pk}_{\mathrm{a}} \sim 7$, sendo que os grupos silanóis podem ser classificados basicamente em duas categorias : livres (isolados) (a) e ligados (ligações de hidrogênio) (b). Além destes, verifica-se também a presença de grupos siloxanos na superfície da sílica, conforme ilustrado na Figura 1 (c).

A desidroxilação da superfície da sílica acontece em mais de uma etapa. Aquecendo-se até aproximadamente $400 \mathrm{~K}$, remove-se basicamente a água fisisorvida. Acima dessa temperatura, inicia-se a condensação dos grupos silanóis, produzindo água $^{2}$, conforme ilustrado na Figura 2.

* endereço atual: Instituto de Química, Unicamp, Caixa Postal 6154, 13083-970, Campinas, São Paulo.e-mail: rffarias@iqm.unicamp.br

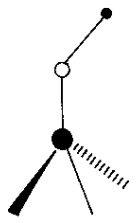

A

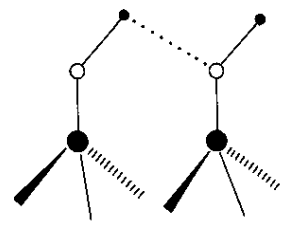

B

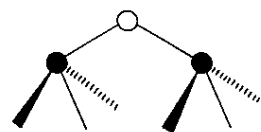

C

$$
\begin{aligned}
& 0=\text { Silicio } \\
& 0=\text { Oxigēnio } \\
& 0=\text { Hidrogēnio }
\end{aligned}
$$

Figura 1. Composição típica da superfície da sílica-gel: A (silanol isolado); B (silanóis vicinais: ligações de hidrogênio); C (grupo siloxano).

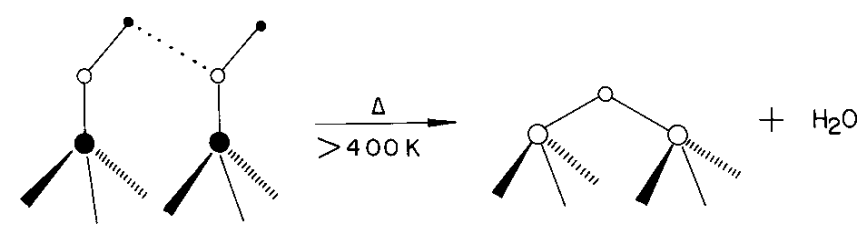

Figura 2. Estequiometria de desidratação da superfície de sílica-gel.

Uma pequena quantidade de grupos hidroxilas livres está sempre presente na superfície da sílica a baixas temperaturas de pré-tratamento. Porém, sob aquecimento a temperaturas próximas a $973 \mathrm{~K}$, a superfície apresenta basicamente grupos hidroxilas livres ${ }^{10}$, sendo que acima de $1073 \mathrm{~K}$, o processo de condensação dos silanóis é irreversível ${ }^{11,12}$. Quando totalmente hidratada a superfície da sílica relembra a face 100 da cristobalita, na qual cada átomo de silício da superfície liga-se a dois grupos $\mathrm{OH}^{13,14}$.

Através do uso da espectroscopia na região do infravermelho com transformada de Fourier (FTIR) com detecção foto-acústica, para estudar-se a modificação química da superfície da sílica com triclorossilano ${ }^{15}$, constatou-se que o mesmo reage exclusivamente com os grupos hidroxilas livres a temperaturas de pré-tratamento (ativação) elevadas, e parcialmente com os grupos hidroxilas ligados, a baixas temperaturas, sendo que a reatividade dos grupos hidroxila livres não é afetada pela diminuição do raio dos poros, enquanto que os grupos ligados entre si por ligações de hidrogênio, mostram-se menos reativos frente ao triclorossilano à medida que o raio dos poros decresce.

Para a maior parte das moléculas adsorvidas, os grupos hidroxila livres costumam mostrar-se como sítios de adsorção mais fortes, sendo que, a baixas pressões, estes sítios são responsáveis 
pela ocorrência da maior parte da adsorção ${ }^{16}$. Os grupos siloxanos podem mostrar-se também como grupos reativos, como por exemplo, na adsorção dissociativa de $\mathrm{B}_{2} \mathrm{H}_{6}{ }^{17}$.

A sílica-gel pode ser sintetizada pura ${ }^{18}, \operatorname{dopada}^{19}$ ou funcionalizada $^{20,21}$, utilizando-se o chamado processo sol-gel, podendo-se inclusive controlar o diâmetro das esferas ${ }^{18}$ e a área superficial da sílica obtida ${ }^{22}$, atingindo-se valores de até 850 $\mathrm{m}^{2} \mathrm{~g}^{-1}$. Pode-se ainda preparar sílica a partir de soluções supersaturadas de ácido silícico ${ }^{23}$, sendo que a desidratação ou a presença de moléculas quimissorvidas exercem efeito marcante sobre as propriedades da sílica-gel ${ }^{24}$.

Quando modificada por um agente organofuncionalizante, a sílica-gel pode ser utilizada como suporte para catalisadores ${ }^{25-27}$, cromatografia $^{28-36}$ ou ainda como agente quelante para a complexação de metais em solução ${ }^{37-45}$, sendo que, no caso de dialquilaminas ligadas linearmente à superfície, o poder sequestrante frente aos metais depende do número de grupos amino primários presentes e não da quantidade total de nitrogênio ${ }^{46}$. É também conhecido o uso de organossilanos para produzir superfícies com propriedades antimicrobianas ${ }^{47}$, para imobilização de enzimas em suportes sólidos ${ }^{48}$ e para adsorver proteínas do sangue ${ }^{49}$.

Através de estudos de fluorescência de raios-X ${ }^{50,51}$, constata-se que moléculas quimicamente ligadas à sílica microparticulada não se encontram distribuídas homogeneamente, mas agrupadas em regiões de alta densidade.

A chamada sililação é o deslocamento de um hidrogênio ativo, usualmente em um grupo hidroxila, por um grupo organosilil. $\mathrm{O}$ agente sililante age como um intermediário capaz de promover a ligação covalente de um material orgânico ou inorgânico à uma superfície mineral, obtendo-se com isso as vantagens da presença de grupos reativos em um substrato insolúvel $^{52}$. Na maior parte das aplicações, é desejável que o agente sililante seja resistente à remoção da superfície por solventes orgânicos ou mesmo água ${ }^{52}$.

Os agentes sililantes, que são todos obtidos sinteticamente ${ }^{53}$, podem ser utilizados para reação com outros suportes inorgânicos diferentes da sílica ${ }^{54,55}$, ou ainda misturados entre si para produzir uma rede polimérica que seja capaz de encapsular enzimas ${ }^{56,57}$, podendo ainda ter sua reatividade induzida por radiação ${ }^{58}$.

Todos os organosilanos comercialmente disponíveis, como por exemplo $\mathrm{X}\left(\mathrm{CH}_{2}\right)_{3} \mathrm{Si}\left(\mathrm{OCH}_{3}\right)_{3}$, com $\mathrm{X}=\mathrm{Cl}, \mathrm{NH}_{2}, \mathrm{SH}$, etc., possuem três grupos reativos ligados ao silício, que são utilizados para promover a ligação covalente à superfície com a qual reagem $^{52}$. Através da utilização de agentes sililantes com diferentes velocidades de reação frente à sílica, pode-se produzir um material com composição superficial diferente daquela existente no interior dos poros ${ }^{59}$. Nas reações com sílica, normalmente as temperaturas são mantidas na faixa de 393-473 K sendo estas condições necessárias para promover a reação entre o alcoxissilano e a superfície ${ }^{60}$ para um tempo de cura de $3 \mathrm{~h}$. Do ponto de vista experimental a temperatura de $353 \mathrm{~K}$ é considerada suficiente para atingir-se a máxima estabilização das ligações entre o alcoxissilano e a superfície da sílica ${ }^{61}$.

Aminas e compostos de estanho são catalisadores para a reação de alcoxissilanos com superfícies minerais. Silanos aminofuncionais são auto-catalisadores, mas alcoxissilanos não aminados são mais facilmente ligados na presença de pequenas quantidades de uma amina orgânica ou de um organosilano aminofuncional ${ }^{52}$.

As fases ligadas obtidas a partir de organossilanos são totalmente imunes à hidrólise, devido à formação de ligações Si-O$\mathrm{Si}^{62}$. Porém, a fim de prevenir a formação de grupos hidroxilas livres nas moléculas dos organossilanos e seus polímeros, a reação entre estes e o substrato deve ser realizada em solventes $\operatorname{secos}{ }^{63-65}$. Contudo, a presença de água adsorvida no substrato durante a reação parece aumentar a reatividade total, particularmente no caso de etoxissilanos ${ }^{66}$.

Na reação entre aminopropiltrietóxisilano, APTS, que é um dos agentes sililantes mais utilizados com sílica, uma monocamada é formada pela rápida adsorção de moléculas do silano na superfície, independentemente da concentração inicial de APTS $^{64}$, sendo que cada molécula deste agente sililante cobre uma área de $0,64 \mathrm{~nm}^{2}$.

Espectroscopias de infravermelho, (FTIR) e reflectância difusa (DRIFT) costumam ser rotineiramente utilizadas para a caracterização da sílica organofuncionalizada ${ }^{65,67-89}$, não apenas para caracterizar a presença das moléculas ancoradas na superfície, mas também para determinações quantitativas ${ }^{90-92}$ ou para o estudo da cinética de reação ${ }^{61}$, $93-96$.

Outra técnica comumente utilizada é a ressonância magnética nuclear CP MAS (Cross Polarization Magic-Angle Spinning) de ${ }^{13} \mathrm{C},{ }^{15} \mathrm{~N}$ e ${ }^{29} \mathrm{Si}^{97-106}$, sendo que RMN de ${ }^{1} \mathrm{H}$ costuma ser utilizada para estudar-se a desidratação da superfície da sílica ${ }^{107-108}$.

A espectroscopia Raman ${ }^{109,110}$,termogravimetria de alta resolução $^{111,112}$, calorimetria exploratória diferencial ${ }^{19}$ (DSC), microscopia eletrônica de varredura ${ }^{113}$, e espectroscopia Auger ${ }^{114}$ são também comumente utilizadas para a caracterização de sílicas. Outras técnicas são ainda empregadas em maior ou menor extensão.

\section{Sílica Lamelar a partir de Silicatos Alcalinos: Ácido Silícico}

Uma rota de síntese comumente utilizada para a obtenção de sílica lamelar, é a síntese de silicatos de sódio ou potássio lamelares ${ }^{115-120}$, promovendo-se, em seguida, a troca iônica do $\mathrm{Na}^{+}$ou $\mathrm{K}^{+}$do silicato cristalino, por íons $\mathrm{H}^{+}$, provenientes de uma solução de $\mathrm{HCl}^{115}$, obtendo-se assim o respectivo ácido silícico. Contudo, esta rota de síntese apresenta algumas dificuldades, tais como tempo de reação prolongado e, principalmente, dificuldades relacionadas à reprodutibilidade de resultados, sendo que muitas sínteses conduzem a produtos com baixa cristalinidade.

Numa síntese típica, sílica amorfa, hidróxido de sódio ou potássio e água, são misturados na seguinte proporção molar: 3 $\mathrm{SiO}_{2}+\mathrm{KOH}+40 \mathrm{H}_{2} \mathrm{O}$. A mistura reacional é aquecida a 398 $\mathrm{K}$ durante 21 dias. $\mathrm{O}$ produto final obtido apresenta fórmula $\mathrm{K}_{2} \mathrm{H}_{2} \mathrm{Si}_{20} \mathrm{O}_{42}$. $\mathrm{xH}_{2} \mathrm{O}$. A troca iônica anteriormente mencionada, é promovida dispersando-se o silicato cristalino obtido em um excesso de uma solução $1,0 \mathrm{~mol} \mathrm{\textrm {dm } ^ { 3 }}$ de $\mathrm{HCl}$. A etapa crítica neste tipo de síntese não é a tranformação do silicato cristalino no respectivo ácido silícico, mas a síntese do próprio silicato, sendo observado que, mesmo utilizando-se iguais condições de síntese, muitas vezes os produtos obtidos diferem em cristalinidade, bem como em reatividade ${ }^{115}$. A obtenção de silicatos alcalinos por esta via de síntese, costuma consumir dezenas de dias, ou mesmo anos ${ }^{115-120}$. Silicatos alcalinos podem também ser preparados através de reações no estado sólido ${ }^{121}$, reduzindo o tempo de síntese para menos de $24 \mathrm{~h}^{121}$.

A estabilidade térmica dos ácidos silícicos assim obtidos costuma diminuir com o aumento da cristalinidade ${ }^{115}$, enquanto que a reatividade é aumentada à medida que a cristalinidade aumenta ${ }^{115}$, podendo-se utilizar calorimetria exploratória diferencial (DSC) ou calorimetria de solução para se determinar o grau de cristalinidade de amostras de sílica ${ }^{122,123}$.

A silinaíta ${ }^{116}, \mathrm{NaLiSi}_{2} \mathrm{O}_{5} .5 \mathrm{H}_{2} \mathrm{O}$, é um silicato de lítio e sódio que pode também ser obtida e convertida no seu respectivo ácido silícico, utilizando-se condições de síntese similares àquelas utilizadas para os silicatos de sódio e potássio. Observa-se que os íons $\mathrm{Li}^{+}$e $\mathrm{Na}^{+}$podem ser trocados por prótons ou íons alquilamônio ${ }^{116}$, sendo que a obtenção da forma ácida, aumenta a área superficial específica do composto $^{116}$. O produto final obtido após a troca dos íons alcalinos por íons $\mathrm{H}^{+}$mostra-se dependente da concentração da solução ácida utilizada, sendo que, geralmente, soluções mais concentradas, e.g. $\mathrm{HNO}_{3} 7,0 \mathrm{~mol} \mathrm{dm}^{3}$ ou $\mathrm{HCl} 6,0 \mathrm{~mol} \mathrm{dm}^{3}$, produzem o respectivo ácido silícico derivado da silinaíta, enquanto que soluções diluídas promovem a transformação da silinaíta em $\alpha-\mathrm{H}_{2} \mathrm{Si}_{2} \mathrm{O}_{5}{ }^{116}$. 
Na silinaíta, a conversão do silicato alcalino em seu respectivo ácido silícico não altera a morfologia dos grãos obtidos, mas modifica significativamente a capacidade do composto de adsorver gases ${ }^{116}$. As amostras obtidas mostram-se relativamente instáveis em suas propriedades, como ilustrado pela mudança de área superficial de uma amostra de $\alpha-\mathrm{H}_{2} \mathrm{Si}_{2} \mathrm{O}_{5}$, passando de $95 \mathrm{~m}^{2} \mathrm{~g}^{-1}$ para $225 \mathrm{~m}^{2} \mathrm{~g}^{-1}$, num período de quatro anos ${ }^{116}$.

De acordo com a estrutura cristalina, todos os átomos de $\mathrm{Si}$ no ácido silícico derivado da silinaíta são quimicamente equivalentes, o que pode ser realmente confirmado por RMN de ${ }^{29} \mathrm{Si}^{116}$. No espectro de infravermelho, a banda larga observada entre $3000 \mathrm{~cm}^{-1}$ e $3600 \mathrm{~cm}^{-1}$, é atribuída à presença de pontes de hidrogênio intra e inter lamelares ${ }^{116}$.

A sílica lamelar na forma de ácido silícico obtida a partir de troca iônica promovida em silicatos alcalinos exibe uma elevada reatividade intracristalina, mostrando-se capaz de funcionar como hospedeiro para a formação de compostos de intercalação envolvendo álcoois, alquil e aril aminas, nitrilas, cetonas e aminoácidos ${ }^{115-120}$. A intercalação geralmente é efetuada em solução, mas pode também acontecer com a matriz do ácido silícico e a espécie química a ser intercalada no estado sólido, como é o caso da intercalação de imidazol na forma ácida da silinaíta ${ }^{116}$.

Os ácidos silícicos de fórmula $\mathrm{H}_{4} \mathrm{Si}_{20} \mathrm{O}_{42} \cdot \mathrm{xH}_{2} \mathrm{O}$ e $\mathrm{H}_{4} \mathrm{Si}_{14} \mathrm{O}_{30} \cdot \mathrm{xH}_{2} \mathrm{O}$ apresentam uma maior reatividade frente aos álcoois à medida que a cristalinidade aumenta ${ }^{115}$, com metanol e etanol sendo adsorvidos preferencialmente ${ }^{115}$. A adsorção de aminas em soluções alcoólicas revela a competição existente entre as aminas e as moléculas dos álcoois pela utilização dos sítios reativos intracristalinos ${ }^{119}$.

$\mathrm{O}$ ácido silícico de fórmula $\mathrm{H}_{2} \mathrm{Si}_{20} \mathrm{O}_{41} \cdot \mathrm{xH}_{2} \mathrm{O}^{117}$, geralmente mantém, em solução, a distância interplanar característica dos silicatos de sódio ou potássio, dos quais pode ser derivado, sendo que após secagem, a distância interplanar costuma diminuir em cerca de $10 \%{ }^{117}$.

A Figura 3 apresenta, de forma esquemática, as etapas envolvidas na síntese e reatividade dos ácidos silícicos.

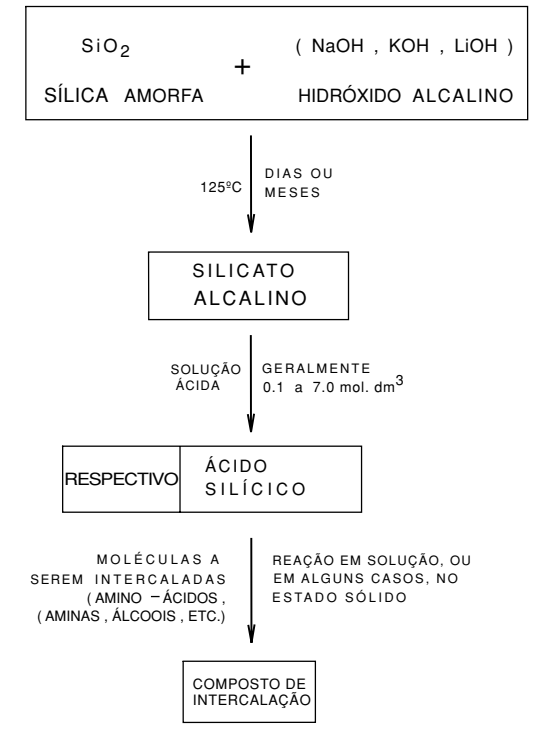

Figura 3. Resumo esquemático da síntese e reatividade dos ácidos silícicos.

\section{Sílica Lamelar Obtida Através do Processo Sol-Gel}

O chamado processo sol-gel ${ }^{124}$ pode ser utilizado para a obtenção de sílica cristalina nas formas lamelar ou hexagonal, utilizando-se alcoxissilanos como precursores e dialquilaminas neutras ${ }^{125,126}$ ou haletos de alquilamônio ${ }^{127-143}$ como moléculas "template", as quais direcionam o processo de polimerização do alcoxissilano. Assim, a polimerização pode ocorrer em direções preferenciais, produzindo um material cristalino, em vez de ocorrer de forma aleatória, o que conduziria à formação de sílica amorfa.

$\mathrm{O}$ uso de baixas temperaturas de síntese, próxima à temperatura ambiente, bem como a rapidez na obtenção dos produtos, uma vez que uma síntese típica consome de alguns minutos até poucas horas, o que certamente é uma vantagem quando comparada por exemplo, com os tempos de síntese geralmente envolvidos na síntese dos ácidos silícicos, tornam o processo sol-gel bastante atraente para aqueles envolvidos com a química das sílicas lamelares. Deve-se lembrar contudo, que a necessidade de espécies orgânicas para atuarem como moléculas "template" torna-se um fator inconveniente, uma vez que, diferentemente dos ácidos silícicos, as sílicas lamelares obtidas por esta rota não apresentam livres os espaços interlamelares. Assim, estas sílicas não se encontram aptas a receberem moléculas hóspedes, já que os espaços interlamelares se mostram ocupados pelas moléculas "template". Estratégias experimentais que propiciem o aproveitamento em termos de reatividade subsequente dos materiais assim obtidos fazem-se necessárias.

Misturas de tetrametóxissilano (TMS) com alquiltrimetoxissilanos de cadeias alquílicas com 6 a 18 carbonos, também conduzem à formação de sílica lamelar ${ }^{144}$. A hidrólise dos alquiltrimetoxissilanos sem a presença de TEOS também pode conduzir à formação de materiais lamelares, sendo que a temperatura de síntese é um fator decisivo. Os valores mais baixos de temperatura conduzem à formação de materiais de maior ordenamento estrutural ${ }^{132}$.

A utilização da chamada rota da dialquilamina neutra para a obtenção de sílica e outros materiais lamelares ganhou destaque com consequente expansão nos últimos cinco anos, principalmente devido à relativa facilidade de obtenção dos materiais, além da possibilidade de, em se variando as condições de síntese, modelar-se os materiais obtidos, no intuíto de controlar suas propriedades.

Na preparação de sílica lamelar utilizando-se o processo solgel, o número de átomos de carbono na cadeia da dialquilamina neutra ou do surfactante e o número de moles de água presentes no sistema reacional são fatores determinantes para que o produto final obtido seja amorfo, hexagonal ou lamelar. Em princípio, cadeias carbônicas maiores e maior quantidade de água, determinam a formação de um produto lamelar, enquanto a reduçao da cadeia carbônica da molécula "template" conduz à formação de material hexagonal ${ }^{125}$. Observa-se que a distância interplanar do produto lamelar obtido, aumenta linearmente com o aumento da cadeia carbônica do surfactante ou da diamina neutra utilizada ${ }^{128,144}$

A Figura 4 apresenta um difratograma de raios-X típico de sílica lamelar. Os três picos observados em $2 \theta$ iguais à $3,8^{\circ}$, $7,3^{\circ}$ e 11,5 devem-se, respectivamente, a picos basais com índices de Miller 001 e 002 e $003^{125,126}$. A Figura 5 apresenta uma micrografia eletrônica de varredura de sílica lamelar, exibindo uma característica estrutura em camadas.

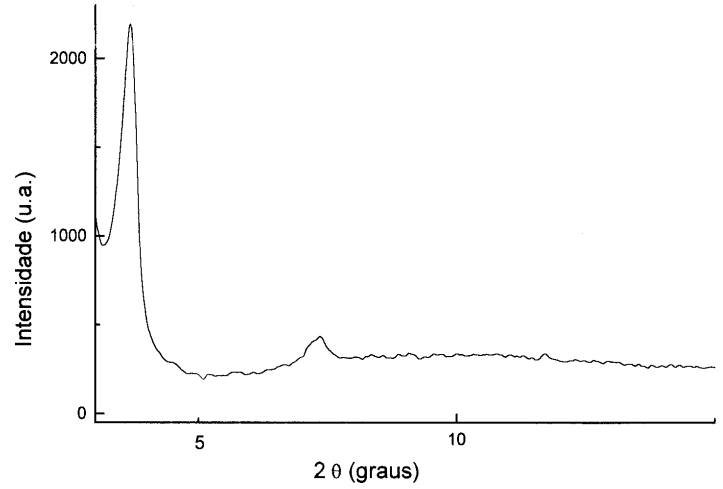

Figura 4. Difratograma de raios-X típico de sílica lamelar obtida pelo processo sol-gel. 


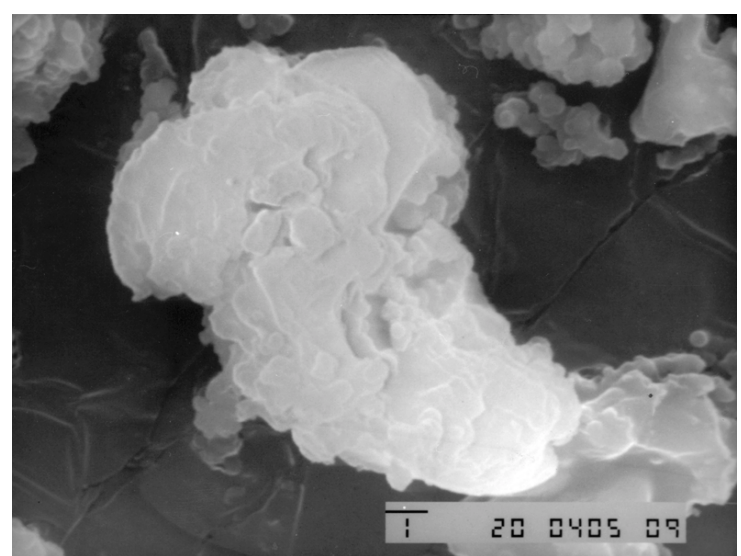

Figura 5. Micrografia eletrônica de varredura de sílica lamelar, exibindo uma típica estrutura em camadas (escala: $-=1 \mu \mathrm{m}$ ).

A sílica lamelar obtida pela chamada rota da dialquilamina neutra converte-se na fase hexagonal ao se remover a diamina mediante calcinação ou extração por solvente ${ }^{125}$. Além disso, a adição de metais de transição ao sistema reacional pode ser utilizada para controlar a cristalinidade da sílica assim obti$\mathrm{da}^{145}$. Assim, ao se reorientarem para propriciar a coordenação, as moléculas da diamina terminam por determinar alterações nas distâncias interplanares ${ }^{145}$. A establilidade térmica dos materiais obtidos aumenta à medida que a quantidade de metal adicionado é também aumentada ${ }^{145}$. A adição de metais à sílica lamelar também influencia grandemente a entalpia de imersão $\left(\Delta \mathrm{H}_{\text {wet }}\right)$, com as sílicas coordenadas apresentando uma entalpia de imersão da ordem de $40 \mathrm{~J} \mathrm{~g}^{-1}$, enquanto que a sílica lamelar pura, obtida pela rota da diamina neutra, apresenta entalpia de imersão praticamente nula ${ }^{146}$.

Utilizando-se moléculas de brometos de alquilamônio com seis a dezesseis carbonos como surfactantes e tetrametilortosilicato (TMOS) como precursor, pode-se obter, através do procedimento de "spin coating" materiais lamelares sob a forma de filmes transparentes ${ }^{128}$, que apresentam as cadeias de surfactantes posicionadas de forma inclinada em aproximadamente $50^{\circ}$ no interior das lamelas ${ }^{128}$. Para os filmes lamelares produzidos utilizando-se este procedimento, verifica-se que uma rápida evaporação do solvente constitui-se em fator fundamental para a formação de matrizes altamente ordenadas ${ }^{133}$. A orientação das cadeias do surfactante no interior das lamelas dependerá da densidade de carga na camada de sílica ${ }^{141}$, sendo que a espessura da camada de sílica aumenta à medida que a relação TMOS/surfactante aumenta ${ }^{133}$. As Figura 6 e 7, ilustram estes dois fenômenos.

Em se tratando de diaminas neutras, a sílica lamelar obtida pode apresentar as cadeias de diamina perpendiculares às camadas de sílica ${ }^{145}$. Porém, com a introdução de metais de transição pode ter, entre outros benefícios, a possibilidade de atuar como estratégia de síntese, a fim de se estimar a distância média entre as cadeias de diamina ${ }^{145,147,148}$.

\section{CONCLUSÃO}

A título de conclusão, deve ser dito que, a obtenção de sílica e outros materiais lamelares utilizando-se o chamado processo sol-gel mostra-se como um caminho bastante promissor para a síntese de materiais com grande potencialidade de aplicação como catalisadores ${ }^{136}$ ou materiais ópticos por exemplo.

A versatilidade do processo sol-gel permitindo a realização de sínteses a baixas temperaturas, e possibilitando a obtenção de materiais híbridos de grande interesse a nível de estudo básico ou aplicado, certamente constituirá, ainda por muitos anos, um excitante campo de estudo a ser explorado.
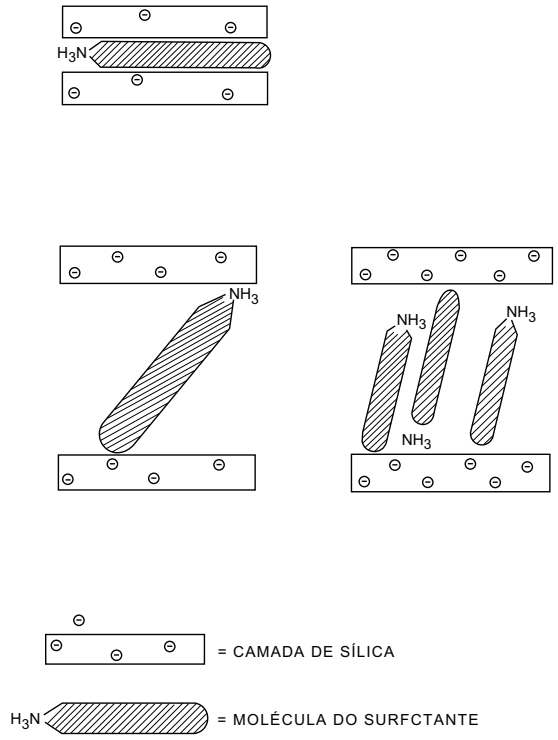

Figura 6. Orientação das cadeias de surfactante no interior da sílica lamelar, em função da distribuição de cargas no substrato.

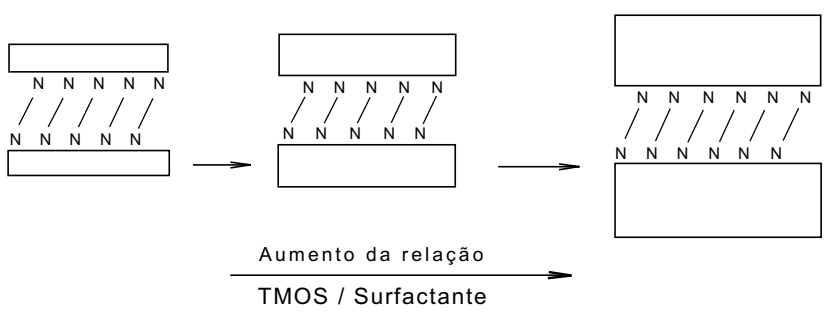

Figura 7. Aumento da espessura da camada de sílica, com o aumento da relação TMOS/surfactante.

\section{AGRADECIMENTOS}

À FAPESP pelo apoio financeiro. À CAPES-PICDT e CNPq pelas bolsas recebidas.

\section{REFERÊNCIAS}

1. Degens, E. D.; in Topics in Current Chemistry vol. 64, Inorganic Biochemistry, Springer-Verlag, New York, 1976.

2. Iler, R. K.; The Chemistry of Silica, John Wiley \& Sons, New York, 1979.

3. Cerrato, G.; Fubini, B.; Baricco, M.; Moterra, C.; J. Mater. Chem. 1995, 5, 1935.

4. Wiessner, J. H.; Mandel, N. S.; Sohnle, P. G.; Hasegawa, A.; Mandel, G. S.; Am. Rev. Resp. Dis. 1990, 141, 111.

5. Wiessner, J. H.; Henderson, J. D. Jr.; Sohnle, P. G., Mandel, N. S.; Mandel, G. S.; Am. Rev. Resp. Dis. 1988, 138,445

6. Kiselev, A. V.; Kollodn. Zh. 1936, 2, 17.

7. Zhuravlev, L. T.; Langmuir 1987, 3, 316.

8. Farias, R. F. de; Airoldi, C.; J. Therm. Anal. no prelo.

9. Gates, B. C.; Catalytic Chemistry, John Wiley \& Sons, New York, 1992.

10. Van Der Voort, P.; Gillis-D'Hamers, I.; Vansant, E. F.; $J$. Chem. Soc. Faraday Trans. 1990, 86, 3751.

11. Lochmuller, C. H.; Kersey, M. T.; Langmuir 1988, 4, 572.

12. Hair, M. L.; in Silanes, Surfaces and Interfaces, Gordon and Breach Science Publishers, New York, 1986.

13. Peri, J. B.; Hensley Jr., A. L.; J. Phys. Chem. 1966, 70, 3941. 14. Carman, P. C.; Trans. Faraday Soc. 1940, 36, 964. 
15. Van Der Voort, P.; Gillis-D'Hamers, I.; Vrancken, K. C.; Vansant, E. F.; J. Chem. Soc. Faraday Trans. 1991, 87, 3899. 16. Hair, M. L.; Hertl,W.; J. Phys. Chem. 1969, 73, 4269.

17. Gillis-D'Hamers, I.; Philippaerts, J.; Van Der Voort, P.;Vansant, E. F.; J. Chem. Soc. Faraday Trans. 1990, $86,3747$.

18. Izutsu, H.; Mizukami, F.; Nair, P. K.; Kiyozumi, Y.; Maeda, K.; J. Mater. Chem. 1997, 7, 767.

19. Dutoit, D. C. M.; Schneider, M.; Fabrizioli, P.; Baiker, A.; J. Mater. Chem. 1997, 7271.

20. Cestari, A. R.; Airoldi, C.; Langmuir 1997, 13, 2681.

21. Ahmad, Z.; Sarwar, M. I.; Mark, J. E.; J. Mater. Chem. 1997, 7, 259.

22. Zehl, G.; Bischoff, S.; Mizukami, F.; Isutzu, H.; Bartoszek, M., Jancke, H.; Lucke, B., Maeda, K.; J. Mater. Chem. 1995, 5, 1893.

23. Makrides, A. C.; Turner, M.; Slaughter, J.; J. Colloid Interf. Sci. 1980, 73, 345.

24. Lowen, W. K.; Broge, E. C.; J. Phys. Chem. 1961, 65, 16.

25. Collman, J. P.; Belmont, J. A.; Brauman, J. I.; J. Am. Chem. Soc. 1983, 105, 7288.

26. Van der Voort, P.; Possemiers, K.; Vansant, E. F.; J. Chem. Soc. Faraday Trans. 1996, 92, 843.

27. Clark, J. H.; Macquarrie, D. J.; Chem. Commun. 1998, 853.

28. Bjorklund, M.; Hearn, M. T. W.; J. Chromatogr. A, 1996, 278,149

29. Lin, C. -E.; Li, F. -K.; Lin, C. -H.; J. Chromatogr. A, 1996, 722, 211.

30. Lork, K. D.; Unger, K. K.; Kinkel, J. N.; J. Chromatogr. 1986, 352, 199 .

31. Karger, B. L.; Sibley, E.; Anal. Chem. 1973, 45, 740.

32. Grushka, E.; Kikta Jr.; E. J.; Anal. Chem. 1974, 46, 1370.

33. Minakuchi, H.; Nakanishi, N.; Soga, N.; Ishizuka, N.;Tanaka, N.; J. Chromatgr. A, 1997, 762, 135.

34. Bjorkhund, M.; Hearn, M. T. W.; J. Chromatgr. A, 1997, $762,113$.

35. Vervoort, R. J. M.; Derksen, M. W. J.; Debets, A. J. J.; J. Chromatogr. A, 1997, 765157.

36. Gilpin, R. K.; Burke, M. F.; Anal. Chem. 1973, 45, 1383.

37. Airoldi, C.; Gonçalves, A. S.; Colloids Surf. 1987, 28, 199.

38. Lishko, T. P.; Glushchenko, L. V.; Kholin, Yu. V.; Zaitsev, Z. N.; Bugaevskii, A. A.; Donskaya, N. D.; Shevchenko, T. G.; Russ. J. Phys. Chem. 1991, 65, 1584.

39. Espínola, J. G. P.; Freitas, J. M. P. de, Oliveira, S. F. de, Airoldi, C.; Colloids Surf. 1994, 87, 33.

40. Alcântara, E. F. C.; Airoldi, C.; Nakamura, O.; Vargas, H.; Paixão, F.; Polyhedron 1992, 11, 1701.

41. Cestari, A. R.; Airoldi, C.; J. Braz. Chem. Soc. 1995, 6, 291.

42. Espínola, J. G. P.; Freitas, J. M. P. de, Oliveira, S. F. de, Airoldi, C.; Colloids Surf. 1992, 68, 261.

43. Airoldi, C.; Alcântara, E. F. C.; Thermochim. Acta 1995 , 259, 95.

44. Airoldi, C.; Alcântara, E. F. C.; J. Chem. Thermodynamics. 1995, 27, 623.

45. Costa, C. M. M.; Pesco, C.; Quím. Nova 1993, 16, 18.

46. Taylor, I.; Howard, A. G.; Anal. Chim. Acta 1993, 271, 77.

47. White, W. C.; Gettings, R. L.; in Silanes, Surfaces and Interfaces, Gordon and Breach Science Publishers, New York, 1986.

48. Chvalovsk_, V.; in Carbon -Functional Organosilicon Compounds, Plenum Press, New York, 1984.

49. Arkles, B.; Lydzinski, D.; Brinigar, W.; Chao, T.; in Silanes, Surfaces and Interfaces, Gordon and Breach Science Publishers, New York, 1986.

50. Lochmuller, C. H.; Colborn, A. S.; Hunnicutt, M. L.; Anal. Chem. 1983, 55, 1344.

51. Lochmuller, C. H.; Colborn, A. S.; Hunnicutt, M. L.; Harris, J. M.; J. Am. Chem. Soc. 1984, 106, 4077.
52. Plueddemann, E. P.; in Silanes, Surfaces and Interfaces, Gordon and Breach Science Publishers, New York, 1986.

53. Tacke, R.; Wannagat, U.; in Topics in Current Chemistry, vol. 84, Bioactive Organo-silicon Compounds, SpringerVerlag, New York, 1979.

54. Moses, P. R.; Wier, L. M.; Lennox, J. C.; Finklea, H. O . Lenhard, J. R.; Murray, R. W.; Anal. Chem. 1978, 50, 576.

55. Seckin, T.; Gultek, A .; Onal, Y.; Yakinci, E.; Aksoy, I.; J. Mater. Chem. 1997, 7265.

56. Venton, D. L.; Clessman, K. L.; Chatterton Jr.; R. T.; Anderson, T. L.; Biochim. Biophys. Acta 1984, 797, 343.

57. Venton, D. L.; Gudipati, E.; in Silanes, Surfaces and Interfaces, Gordon and Breach Science Publishers, New York, 1986.

58. Lampe, F. W.; Snyderman, J. S.; Johnston, W. H.; J. Phys. Chem. 1966, 70, 3934.

59. Williams, D. L.; Tangney, T. J.; in Silanes, Surfaces and Interfaces, Gordon and Breach Science Publishers, New York, 1986.

60. Hertl, W.; J. Phy. Chem. 1968, 72,1248.

61. Waddell, T. G.; Leyden, D. E.; de Bello, M. T.; J. Am. Chem. Soc. 1981, 103, 5303.

62. Grushka, E.; Kikta Jr.; E. J.; Anal. Chem. 1977, 49, 1004 A.

63. Vrancken, K. C.; Casteleyn, E.; Possemiers, K.; Van Der Voort, P.; Vansant, E. F.; J. Chem. Soc. Faraday Trans. 1993, 89, 2037.

64. Vrancken, K. C.; Van Der Voort, P.; Gillis-D'Hamers, I.; Vansant, E. F.; Grobet, P.; J. Chem. Soc. Faraday Trans. 1992, 88, 3197.

65. Sindorf, D. W.; Maciel, G. E.; J. Am. Chem. Soc. 1983 , 105, 3767.

66. Morrow, B. A.; Cody, I. A.; J. Phys. Chem. 1973, 77, 1465.

67. Zhdanov, S. P.; Langmuir 1987, 3, 960.

68. Peri, J. B.; J. Phys. Chem. 1966, 70, 3168.

69. Boccuzzi, F.; Coluccia, S.; Ghiotti, G.; Morterra, C.; Zecchina, A.; J. Phys. Chem. 1978, 82, 1298.

70. Morrow, B. A.; Cody, I. A.; Lee, L. S. M.; J. Phys. Chem. 1976, 80, 2761.

71. Morrow, B. A.; Cody, I. A.; Lee, L. S. M.; J. Phys. Chem. 1975, 79, 2405

72. Ghiotti, G.; Garrone, E.; Morterra, C.; Boccuzzi, F.; J. Phys. Chem. 1979, 83, 2863.

73. Koenig, J. L.; in Silanes, Surfaces and Interfaces, Gordon and Breach Science Publishers, New York, 1986.

74. Bermudez, V. M.; J. Phys. Chem. 1971, 75, 3249.

75. Burneau, A.; Barris, O.; Gallas, J. P.; Lavalley, J. C.; Langmuir 1990, 6, 1364.

76. Tsuchiya, I.; J. Phys. Chem. 1982, 86, 4107.

77. Davydov, V. Ya.; Kiselev, A. V.; Zhuravlev, L. T.; Trans. Faraday Soc. 1964, 60, 2254.

78. Ishikawa, T.; Matsuda, M.; Yasukawa, A.; Kandori, K.; Inagaki, S.; Fukushima, T.; Kondo, S.; J. Chem. Soc. Faraday Trans. 1996, 92, 1985.

79. Benesi, H. A.; Jones, A. C.; J. Phys. Chem. 1959, 63, 179.

80. Snyder, L. R.; Ward, J. W.; J. Phys. Chem. 1966, 70, 3941.

81. Boerio, F. J.; Armogan, L.; Cheng, S. Y.; J. Colloid Interf. Sci. 1980, 73, 416.

82. Peri, J. B.; J. Phys. Chem. 1966, 70, 2937.

83. Moulson, A. J.; Roberts, J. P.; Trans. Faraday Soc. 1961 57, 1208.

84. Hockey, J. A.; Pethica, B. A.; Trans. Faraday Soc. 1961, 57, 2247.

85. McDonald, R. S.; J. Am. Chem. Soc. 1957, 79, 850.

86. Ishida, H.; Koenig, J. L.; J. Colloid. Interf. Sci. 1978, 64, 565.

87. Ishida, H.; Koenig, J. L.; J. Colloid Interf. Sci. 1978, 64, 555.

88. Murthy, R. S. S.; Blitz, J. P.; Leyden, D. E.; Anal. Chem. 1986, 58, 3167.

89. Murthy, R. S. S.; Leyden, D. E.; Anal. Chem. 1986, 58, 1229. 90. Fripiat, J. J.; Uytterhoeven, J.; J. Phys. Chem. 1962, 66, 800. 
91. Fripiat, J. J.; Gastushe, M. C.; Brichard, R.; J. Phys. Chem. 1962, 66, 805.

92. Hair, M. L.; Hertl, W.; J. Phys. Chem. 1973, 77, 2070.

93. Ballard, C. C.; Broge, E. C.; Iler, R. K.; St. John, D. S., McWhorter, J. R.; J. Phys. Chem. 1961, 65, 20.

94. Hertl, W.; Hair, M. L.; J. Phys. Chem. 1971, 75, 2181.

95. Galkin, G. A.; Kiselev, A. V.; Lygin, V. I.; Trans. Faraday Soc. 1964, 60, 431.

96. Carvajal, G. S.; Leyden, D. E.; Quinting, G. R.; Maciel, G. E.; Anal. Chem. 1988, 60, 1776.

97. Sindorf, D. W.; Maciel, G. E.; J. Phys. Chem. 1983, 87, 5516.

98. Sindorf, D. W.; Maciel, G. E.; J. Am. Chem. Soc. 1983, $105,1487$.

99. Sindorf, D. W.; Maciel, G. E.; J. Phys. Chem. 1982, 86, 5208.

100. Maciel, G. E.; Sindorf, D. W.; J. Am. Chem. Soc. 1980, 102, 7606.

101. Chuang, I. -S.; Maciel, G. E.; J. Am. Chem., Soc. 1996, $118,401$.

102. Fyfe, C. A.; Zhang,Y.; Aroca, P.; J. Am. Chem. Soc. 1992, $114,3252$.

103. Kraus, A.; Schneider, M.; Gugel, A.; Mullen, K.; J. Mater. Chem. 1997, 7, 763.

104. Fyfe, C. A.; Gobbi, G. C.; Kennedy, G. J.; J. Phys. Chem. 1985, 89, 277.

105. Murthy, R. S.; Carvajal, G. S.; Leyden, D. E.; in Silanes, Surfaces and Interfaces, Gordon and Breach Science Publishers, New York, 1986.

106. Bronnimann, C. E.; Zeigler, R. C.; Maciel, G. E.; J. Am. Chem., Soc. 1958, 110, 2023.

107. Zimmerman, J. R.; Lasater, J. A.; J. Phys. Chem. 1958, $62,1157$.

108. Simpson, S. J.; Harris, J. M.; J. Phys. Chem. 1990, 94, 4649.

109. Nikiel, L.; Zerda, T. W.; J. Phys. Chem. 1991, 95, 4063.

110. Nikiel, L.; Hopkins, B.; Zerda, T. W.; J. Phys. Chem. 1990, 94, 7458.

111. Goworek, J.; Stefaniak, W.; Thermochim. Acta 1996, $286,199$.

112. Staszczuk, P.; Jaraniec, M.; Gilpin, R. K.; Thermochim. Acta 1996, 287, 225.

113. Jethmalani, J. M.; Ford, W. T.; Langmuir 1997, 13, 3338.

114. Cain, J. F.; Sacher, E.; J. Colloid. Interf. Sci. 1978, 67, 538.

115. Döring, J.; Lagaly, G.; Beneke, K.; Dékàny, I.; Colloids Surf. A, 1993, 71, 219.

116. Beneke, K.; Thiesen, P.; Lagaly, G.; Inorg. Chem. 1995, 34, 900 .

117. Beneke, K.; Lagaly, G.; Am. Meneral. 1983, 68, 818.

118. Beneke, K.; Kruse, H. -H.; Lagaly, G.; Z. Anorg. Allg. Chem. 1984, 518, 65.

119. Döring, J.; Lagaly, G.; Clay Miner. 1993, 28, 39.

120. Beneke, K.; Lagaly, G.; Am. Miner. 1989, 74, 224.
121. Crone, I. A.; Franklin, K. R.; Graham, P.; J. Mater. Chem. 1995, 5, 2007.

122. Mikhail, S. A.; Turcotte, A. -M.; Thermochim. Acta 1997, 292, 111.

123. Takei, T.; Eriguchi, E.; Fuji, M.; Watanabe, T.; Chikazawa, M.; Thermochim. Acta 1998, 308, 139.

124. Brinker, C. J.; Scherer, G. W.; Sol-Gel Science, Academic Press, New York, 1990.

125. Ulagappan, N.; Battaram, N.; Raju, V. N.; Rao, C. N. R.; Chem. Commun. 1996, 2243.

126. Tanev, P. T.; Pinnavaia, T. J.; Science 1996, 271, 1267.

127. Monnier, A.; Schüth, F.; Huo, Q.; Kumar, D.; Margolese, D.; Maxwell, R. S.; Stucky, G. D.; Krishnamurty, M.; Patroff, P.; Firouzi, A.; Janicke, M.; Chmelka, B. F.; Science 1993, 261, 1299.

128. Ogawa, M.; J. Am. Chem. Soc. 1994, 116, 7941.

129. Hillhouse, H. W.; Okubo, Tatsuya, van Egmond, J. W.; Tsapatsis, M.; Chem. Mater. 1997, 9, 1505.

130. He, J.; tse, J. S.; Klug, D. D.; Preston, K. F.; J. Mater. Chem. 1998, 8, 705.

131. Ogawa, M.; Igarashi, T.; Kuroda, K.; Chem. Mater. 1998 , $10,1382$.

132. Shimojima, A.; Sugahara, Y.; Kuroda, K.; Bull. Chem. Soc. Jpn. 1997, 70, 2847.

133. Ogawa, M.; Igarashi, T.; Kuroda, K.; Bull. Chem. Soc. Jpn, 1997, 70, 2833.

134. Zhou, H. S.; Sasabe, H.; Honma, I.; J. Mater. Chem. 1998, $8,515$.

135. Ogawa, M.; Langmuir 1997, 13, 1853.

136. Lim, M. H.; Blanford, C. F.; Stein, A.; Chem. Mater. 1998, 10, 467.

137. Krishnamoorti, R.; Vaia, R. A.; Giannelis, E. P.; Chem. Mater. 1996, 8, 1728.

138. Ogawa, M.; Langmuir 1995, 11, 4639.

139. Vaia, R. A.; Teukolsky, R. K.; Giannelis, E. P.; Chem. Mater. 1994, 6, 1017.

140. Dubois, M.; Gulik-Krzywicki, Th.; Cabane, B.; Langmuir 1993, 9, 673.

141. Ogawa, M.; Kuroda, K.; Bull. Chem. Soc. Jpn. 1997, 70, 2593.

142. Matijasic, A.; Voegtlin, A. -C.; Patarin, J.; Guth, J. L.;Huve, L.; Chem. Commun. 1996, 1123.

143. Anderson, M. T.; Martin, J. E.; Odinek, J. G.; Newcomer, P. P.; Chem. Mater. 1998, 10, 1490.

144. Shimojima, A.; Sugahara, Y.; Kuroda, K.; J. Am. Chem. Soc. 1998, 120, 4528 .

145. Farias, R. F. de; Airoldi, C.; IX Brazilian Meeting on Inorganic Chemistry, Angra dos Reis, RJ, 1998.

146. Farias, R. F. de; Airoldi, C.; resultados não publicados.

147. Farias, R. F. de; Airoldi, C.; J. Solid State Chem., no prelo.

148. Farias, R. F. de; Airoldi, C.; J. Non-Cryst. Solids, no prelo. 